\title{
SOROEPIDEMIOLOGIA PARA O VÍRUS DA HEPATITE B (VHB) EM GESTANTES/PARTURIENTES E SUA TRANSMISSÃO PARA RECÉM-NASCIDOS EM GOLÂNIA-GO
}

\author{
Divina das Dores P. Cardoso, Edson Luis de Faria, Marli S. Pereira de \\ Azevedo, Divina Aparecida O. Queiroz, Regina Maria B. Martins, \\ Terezinha Teixeira de Souza, Roberto Ruhman Daher e \\ Celina Maria Turchi Martelli
}

\begin{abstract}
Foram coletadas, entre março de 1990 e julbo de 1992, 1459 amostras sanguineas de mulheres gestantes/parturientes na cidade de Goiânia-GO, objetivando detecção da infecção pelo virus da bepatite B (VHB), através dos marcadores sorológicos AgHBs e anti-HBs. O percentual de positividade encontrado, pelo teste imunoenzimático, foi de $7,5 \%$, sendo $0,5 \%$ para $A g H B$ s e 7,0\% para anti-HBs. A análise efetuada, considerando a faixa etária, mostra que 7 de 8 mulheres AgHBs-positivas pertenciam à faixa etäria de até 30 anos, situação semelbante em relação ao anti-HBs(83/101). Das 8 mulberes positivas, 4 tiveram seus recém-nascidos submetidos a tratamento profilático com vacina (Engerix B) e imunoglobulina bumana anti-hepatite B (HBIG). Além disso, 3 dessas crianças foram analisadas sorologicamente, sendo que uma era AgHBs-positiva ao nascimento. Doença sexualmente transmissinel e transfusão sanguinea foram fatores de risco que correlacionaram significantemente com a infecção. Esses resultados parece-nos reforçar a indicação de triagem à infecção pelo virus da bepatite $B$ no periodo pré-natal, assim como a adoção de medidas iniunoprofiláticas nas crianças nascidas de mães positivas.
\end{abstract}

Palavtas-chaves: Hepatite B. Gestante. Recém-nzascido.

A hepatite por vírus é problema importante em saúde pública, tanto em paises desenvolvidos quanto em desenvolvimento, e a ocasionada pelo vírus B (VHB), é considerado condição grave, não só em termos de prevalência mas, principalmente, pelas conseqüências da infecção, que têm resultado em 300 milhões de pessoas portadoras do vírus em todo o mundo ${ }^{17}$.

Dentre as diferentes formas de transmissão do vírus, destaca-se a vertical ${ }^{25}$, e estima-se que responda por 30,0 a $40,0 \%$ dos portadores crônicos ${ }^{12}$, além de reflexos maiores, como

\footnotetext{
Laboratório de Virologia/Departamento de Microbiologia/Instituto de Patologia Tropical e Saúde Pública/Universidade Federal de Goiás, Goiânia, GO.

Suporte Financeiro: CNPq Processo $\mathrm{n}^{\circ}$ 400641-90.8, CONCITEG (Conselho de Ciência e Tecnologia do Estado de Goiás), PNI (Plano Nacional de Imunização).

Endereço para correspondência: Prof Divina das Dores de Paula Cardoso. R. Delenda Rezende de Melo, esquina com $1^{2}$ avenida, Setor Universitário, 74605-050 Goiânia, GO. Recebido para publicação em 17/07/95.
}

cirrose e carcinoma hepatocelular primário ${ }^{38}$

Triagem sorológica para o VHB em mulheres gestantes tem sido recomendada ${ }^{6}$ na rotina do pré-natal, desde que tratamento profilático de recém-nascido com imunoglobulina humana anti-hepatite B e vacina, diminui sensivelmente o percentual de portadores $271216 \mathrm{e}$ conseqüentemente, a transmissão viral.

Este estudo apresenta informaçôes referentes a análise sorológica para VHB em 1459 mulheres gestantes/parturientes de Goiânia$\mathrm{GO}$, além do monitoramento de 4 crianças nascidas de mães positivas, dados esses que são únicos em nossa região, nesse tipo populacional.

\section{MATERIAL E MÉTODOS}

População estudo. No período de março de 1990 a julho de 1992, foram coletadas em dois hospitais públicos de Goiânia-GO, (Horpital Geral de Goiânia-INAMPS e Hospital Materno Infantil-SSGO), amostras sanguíneas de 1459 mulheres gestantes/parturientes objetivando detecção de marcadores do vírus da hepatite $\mathrm{B}$ 
Cardoso DDP, Faria EL, Azevedo MSP, Queiroz DAO, Martins RMB, Souza TT, Daber RR, Martelli CMT. Soroepidemiologia para vírus da bepatite $B$ (VHB) em gestantes/parturientes e sua transmissāo para recémnascidos em Goiânia, GO. Revista da Sociedade Brasiletra de Medicina Tropical 29:349-353, jul-ago, 1996.

(VHB). A participação no estudo foi voluntária; com consentimento assinado por cada mulher. De cada uma delas foi inquirido dados de ordem pessoal e fatores de risco para a infecção como contato sexual extraconjugal, presença de tatuagem, transfusão sanguínea, doença sexualmente transmissivel além de história prévia de hepatite.

A população estudada situava-se na faixa etária de 12 a 49 anos. Apresentava precária condição sócio-econômico-cultural, observada dentre outros fatores por 59,9\% terem renda familiar inferior a 2 salários mínimos e $75,1 \%$ com nível de escolaridade de ${ }^{\circ}$ grau incompleto.

Metodologia. A coleta do sangue foi feita em uma única ocasião, quando a gestante se internava para o parto, por punção venosa. Todas as amostras foram triadas pelos marcadores AgHBs e anti-HBs. Aquelas positivas para AgHBs foram também testadas para AgHBe, anti-HBe, anti-HBc-total e anti$\mathrm{HBc}-\mathrm{IgM}$. A pesquisa para AgHBs foi feita imediatamente após a coleta, e se havia positividade, se tentava a permissão da mãe para a inclusão do recém-nascido no estudo, e assim, 3 de 4 crianças foram amostradas e analisadas para todos os marcadores. Todas as crianças foram avaliadas clinicamente $e$ tratadas profilaticamente com imunoglobulina humana anti-hepatite B (HBIG-Abbot laboratórios do Brasil), em dose única ao nascer $(0,5 \mathrm{ml})$ e vacina (Engerix-B $-10 \mu \mathrm{g} / \mathrm{ml}$ ), em 3 doses $\left(0,1^{\circ}\right.$ e $6^{\circ}$ mês de vida). A aplicação foi feita por via intramuscular, em coxas distintas. As vacinas foram doadas pelo Ministério da Saúde - Plano Nacional de Imunização.

A metodologia utilizada na análise de todos os marcadores foi o ensaio imunoenzimático (ELISA), segundo Voller e col, 1978 ${ }^{\text {is }}$. Para execução dos experimentos de AgHBs, antiHBs e anti-HBc-IgM foram utilizados imunobiológicos preparados e cedidos pelo Centro de Referência Nacional em Hepatites Virais-Fundação Oswaldo Cruz. Para o antiHBc-total, AgHBe e anti-HBe utilizaram-se imunobiológicos comerciais da Organon Teknica (Hepanostika).

Análise estatística. Para análise comparativa dos resultados, foram utilizados o teste do $\chi_{2}^{2}$ com intervalo de confiança de $95,0 \%$, e $\chi^{2}$ para tendência.

\section{RESULTADOS}

A Tabela 1 mostra que de 1459 mulheres gestantes/parturientes, $8(0,5 \%)$ eram AgHBs positivas e $101(7,0 \%)$ apresentavam anti-HBs. Também, $1301(89,2 \%)$ mulheres estavam na faixa etária de até 30 anos inclusive $7(87,5 \%)$, das 8 mulheres positivas ao AgHBs $(p>0,05)$. Quanto ao anti-HBs, o percentual de soropositividade foi significantemente crescente com o aumento da faixa etária $(\mathrm{p}<0,01)$.

Tabela 1 - Soropositividade aо VHB, pelos marcadores AgHBs e anti-HBs. em mulberes gestantes/parturientes de Goiânia-GO, por faixa etäria.

\begin{tabular}{|c|c|c|c|c|}
\hline \multirow{3}{*}{$\frac{\text { Faixa etária(anos) }}{<20}$} & \multicolumn{2}{|c|}{ AgHBs* } & \multirow{2}{*}{\multicolumn{2}{|c|}{$\begin{array}{c}\text { Anti-HBs** } \\
\text { № pos. } / n^{\circ} \text { exam. } \%\end{array}$}} \\
\hline & $\mathrm{N}^{\mathrm{g}} \mathrm{pos} . / \mathrm{n}$ & xam.\% & & \\
\hline & $4 / 556$ & 0,7 & $28 / 556$ & 5,0 \\
\hline $20-29$ & $3 / 745$ & 0,4 & $55 / 745$ & 7,3 \\
\hline $30-39$ & $1 / 149$ & 0,6 & $16 / 149$ & 10.7 \\
\hline$>40$ & $0 / 9$ & - & $2 / 9$ & 22,2 \\
\hline Total & $8 / 1459$ & 0,5 & $101 / 1459$ & 0,7 \\
\hline
\end{tabular}

O perfil sorológico das 8 mulheres positivas ao AgHBs é evidenciado na Tabela 2. Todas eram anti-HBc-total positivas e anti-HBs negativas e ainda, 3/eram positivas para anti$\mathrm{HBC}-\mathrm{IgM}, 1$ para AgHBe e 4 para anti-HBe.

\begin{tabular}{|c|c|c|c|c|c|}
\hline \multirow[t]{2}{*}{ Paciente } & \multicolumn{5}{|c|}{ Marcadores } \\
\hline & Anti-HBs & Anti-HBc-IgM & Anti-HBc-Total & AgHBe & Anti-HBe \\
\hline$\overline{1}$ & - & - & + & + & - \\
\hline 2 & - & - & + & - & + \\
\hline 3 & - & - & + & - & + \\
\hline 4 & - & - & + & - & - \\
\hline 5 & - & + & + & - & + \\
\hline 6 & - & - & + & - & + \\
\hline 7 & - & + & + & . & - \\
\hline 8 & - & + & + & - & - \\
\hline
\end{tabular}

A Tabela 3 relaciona soropositividade a fatores de risco à infecção. De 109 mulheres positivas ao VHB, $62(56,9 \%)$ admitiram algum fator de risco, sendo que doença sexualmente transmissível (DST) e transfusão sanguínea se relacionaram significantemente, $(\mathrm{p}<0,05) \mathrm{com}$ a soropositividade.

O percentual de mulheres positivas ao VHB, em relação à admissão de história pessoal 
Cardoso DDP, Faria EL, Azevedo MSP, Queiroz DAO, Martins RMB, Souza TT, Daber RR, Martelli CMT. Soroepidemiologia para vírus da bepatite B (VIB) em gestantes/parturientes e sua transmissão para recémnascidos em Goiânia, GO. Revista da Sociedade Brasileira de Medicina Tropical 29:349-353, jul-ago, 1996.

prévia de hepatite, é indicado na Tabela 4. De 109 mulheres positivas, $40(36,7 \%)$ declararam ter tido hepatite prévia e, dessas, apenas 4 $(10,4 \%)$ tinham algum marcador para a infecção pelo vírus, o que dá um valor preditivo positivo (VPP) de $10,0 \%$ e valor preditivo negativo (VPN) de $92,7 \%$.

Tabela 3 - Percentual de mutheres positivas ao VHB (AgHBs ou anti-HBsj en relação a fatores de risco.

\begin{tabular}{|c|c|c|}
\hline \multirow[b]{2}{*}{ Fatores de risco } & \multicolumn{2}{|c|}{$\mathrm{N}^{2}$ positivos $/ \mathrm{N}^{2}$ examinados } \\
\hline & $\mathrm{N}^{2}$ & $\%$ \\
\hline Promiscuidade sexual & $29 / 352$ & 8,2 \\
\hline História de DST & $8 / 63^{*}$ & 12,7 \\
\hline Transfusão sanguínea & $19 / 145^{*}$ & 13,1 \\
\hline Tatuagem & $6 / 66$ & 9,0 \\
\hline Nenhum & $47 / 833$ & 5.6 \\
\hline Total & $109 / 1459$ & 7,5 \\
\hline
\end{tabular}

${ }^{*} \mathrm{p}<0,05$.

Tabela 4-Percentual de mulberes positivas ao VHB (AgHBs ou anti-HBsj en relação a bistória pessoal prévia de bepatite.

\begin{tabular}{lcc}
\hline Condição & № positivas/ $N o$ examinadas \\
\cline { 2 - 3 } & $\mathrm{N}^{\mathbf{2}}$ & $\%$ \\
\hline Com história & $4 / 40$ & 10,4 \\
Sem história & $105 / 1419$ & 7,0 \\
\hline Total & $109 / 1459$ & 7,5 \\
\hline
\end{tabular}

$\overline{\mathrm{VPP}}-10,0 \% ; \overline{\mathrm{VPN}}-92,7 \%$

Pela Tabela 5 observam-se as condições clínico-laboratoriais dos 4 recém-nascidos submetidos à imunoprofilaxia. Todos tinham boa condição física ao nascer e de 3 analisados sorologicamente, 1 tinha AgHBs e anti-HBctotal e outro era também anti-HBc-total e antiHBe-positivo.

Tabela 5-Condicões clínico-laboratoriais de 4 recém-nascidos de maes AgHBs positivas tratados profilaticamente*.

\begin{tabular}{lcccc}
\hline & \multicolumn{4}{c}{ Crianças } \\
\cline { 2 - 5 } Condições & 1 & 2 & 3 & 4 \\
\hline Peso nascimento & $3150 \mathrm{~g}$ & $2900 \mathrm{~g}$ & $1950 \mathrm{~g}$ & $1800 \mathrm{~g}$ \\
Nascimento a termo & $\mathrm{S}$ & $\mathrm{S}$ & $\mathrm{N}$ & $\mathrm{N}$ \\
Ictericia & $\mathrm{N}$ & $\mathrm{N}$ & $\mathrm{N}$ & $\mathrm{N}$ \\
Má-formação & $\mathrm{N}$ & $\mathrm{N}$ & $\mathrm{N}$ & $\mathrm{N}$ \\
Sorologia & & & & \\
AgHBs & - & $\mathrm{NF}$ & + & - \\
anti-HBs & - & $\mathrm{NF}$ & - & - \\
anti-HBc-total & + & $\mathrm{NF}$ & + & - \\
anti-HBc-IgM & - & $\mathrm{NF}$ & - & - \\
AgHBe & - & $\mathrm{NF}$ & - & - \\
anti-HBe & + & $\mathrm{NF}$ & - & - \\
\hline
\end{tabular}

- $\gamma$-globulina especifica hiperimune e vacina.

$\mathrm{S}=\operatorname{sim} ; \mathrm{N}=$ não; $\mathrm{NF}=$ näo feito.

\section{DISCUSSÃO}

A transmissão vertical do VHB é considerada bastante eficiente, e dependendo do estado infectivo da mãe, pode chegar a taxas tão elevadas quanto $90 \% \%^{5}{ }^{17}$. Dados provenientes de países de alta endemicidade indicam que, aproximadamente, um quarto de recém-nascidos tem alta probabilidade de chegar a óbito5.

Neste estudo, observamos um percentual de soropositividade ao AgHBs de 0,5\% em mulheres gestantes/parturientes. Outros estudos por nós realizados, indicam indices de 1,3\%, $1,9 \%$ e $2,0 \%$ em profissionais de saúde ${ }^{1}$, prisioneiros e primodoadores ${ }^{13}$, e em meninos $\mathrm{de} / \mathrm{na} \mathrm{rua}^{15}$ respectivamente, indicando que nossa regiâo pode ser considerada de baixa a média endemicidade em relação à condição de portadores do vírus, dependendo do tipo populacional estudado. No entanto, quando consideramos a prevalência viral, em relação ao anti-HBs, observamos o indice de $7,0 \%$ e em outras populações, com auxílio de outros marcadores, observamos índices de até $21,4 \%^{1}$, o que sugere uma circulação do vírus bastante significativa em nossa região.

Observamos ainda que a positividade ao marcador AgHBs, situou-se na faixa etária de até 30 anos, de maior atividade sexual, e concordante com dados da literatura, reforçando ser essa atividade fator importante de transmissão viral ${ }^{14}$. O mesmo foi evidenciado em nosso estudo onde doença sexualmente transmissível, a qual em geral é associada a intensa atividade e promiscuidade sexual, mostrou indice significante de soropositividade.

Dentre outros fatores de risco à infecção, observamos que $145(10,0 \%)$ das mulheres haviam se submetido à transfusão de sangue em pelo menos uma ocasião, o que evidencia ser alta a indicação de transfusão sanguínea neste tipo populacional, e destas, 19 (13,1\%) eram positivas ao virus, o que se constituiu em fator de risco significante $(p<0,05)$ para a soropositividade viral.

Quando consideramos a história prévia de hepatite, verificamos que apenas $10,4 \%$ das mulheres positivas relataram essa condição, $O$ que forneceu um VPP de 10,0\% e VPN de $92,7 \%$, corroborando o fato de que a infecção é na maioria dos casos inaparente ${ }^{11}$, o que contribui para o aumento da disseminação viral. 
Cardoso DDP, Faria EL, Azevedo MSP, Queiroz DAO, Martins RMB, Souza TT, Daber RR, Martelli CMT. Soroepidemiologia para virus da bepatite B (VHB) em gestantes/parturientes e sua transmissão para recémnascidos em Goiânia, GO. Revista da Sociedade Brasileira de Medicina Tropical 29:349-353, jul-ago, 1996.

Dentre as 8 murtheres positivas para AgHBs, 3 também o eram para anti-HBc-IgM, e outra para AgHBe, condições essas que sugerem multiplicação viral, com probabilidade de transmissão aumentada. Por outro lado, a análise sorológica do sangue do cordão umbilical, de três recém-nascidos, mostrou que 2 deles apresentavam marcadores para o vírus. Destes, um era AgHBs positivo, ambos eram anti-HBctotal positivos, e um deles era ainda, anti-HBe positivo. Embora sem continuação prospectiva deste estudo, podemos conjeturar que estes marcadores não sejam resultado de infecçâo transplacentária, mas que possam ser oriundos de contaminação in útero ou ao nascer', uma vez que não houve detecção de anti-HBc-IgM, - que demonstra não existir uma resposta imune primária ao vírus, originária das crianças.

Procedemos o tratamento profilático de 4 crianças, acreditando ser este um procedimento correto, pois na ausência de qualquer medida profilática, as crianças têm $85,0 \%$ de chance de se tornarem portadoras crônicas, com evolução para cirrose e ou carcinoma hepatocelular ${ }^{3}$. Desta forma sugerimos, independente de critérios de alto risco para infecção e em concordância com a literatura ${ }^{410}$, que triagem para o VHB deva ser implementada na rotina do pré-natal, bem como administração de imunoglobulina humana anti-hepatite $B$ (HBIG) e vacina, em todos os casos de mães sorologicamente positivas, o que por certo contribuirá para limitação do espalhamento da infecção pelo vírus B e agente Delta.

\section{SUMMARY}

In order to detect bepatitis B virus (HBV), 1459 serum samples from pregnant/parturient women were collected at two public hospitals in Goiânia, GO. These samples were tested by enzyme linked immunosorbent assay for HBsAg and anti-HBs. 109 (7.5\%) serum samples were positive. Eight (0.5\%) sera were positive for HBsAg and 101 (7.0\%) for anti-HBs. Viral positivity for botb HBsAg and anti$H B s$ were observed in women which age ranged from 15 to 30 years. Four newborns from HBsAg positive mothers were submitted to the treatment with $H B V$ vaccine (Engerix B) and with byperimmune gammaglobulin ( $H B I G$ - Abbott Laboratories - Brazil). Cord blood from one of the newborns was positive for HBsAg. A positive association was found between hepatitis $B$ and sexually transmited infections and blood transfusion. These results emphasize the need for prenakat screentizg for HBV in pregnant women and treatment of the newborns from $\mathrm{AgHBS}$ positive mothers.

Key-words: Hepatitis B. Pregnant women. Newborn.

\section{REFERÊNCIAS BIBLIOGRÁFICAS}

1. Azevedo MSP, Cardoso DDP, Martins RMB, Daher RR, Camarota SCT, Barbosa AJ. Rastreamento sorológico para hepatite $B$ em profissionais de saúde na cidade de Goiânia, GO. Revista da Sociedade Brasileira de Medicina Tropical 27:157162, 1994.

2. Beasley RP, Hwang Lu-Yu. Postnatal infectivity of hepatitis B surface antigen-carrier mothers. The Journal of Infectious Diseases 147:185-190, 1983.

3. Beasley RP, Shiao I-S, Wu T-C, Hwang LY. Hepatoma in an HBsAg carrier - Seven years after perinatal infection. The Journal of Pediatrics 101:83-84, 1982.

4. Bogomolski-Yahalom V, Granot E, Linder N, Adler R, Korman S, Manny N, Tur-Kaspa R, Shouval D. Prevalence of HBsAg carriers in native and immigrant pregnant female populations in Israel and passive/active vaccination against $\mathrm{HBV}$ of newborns at risk. Journal of Medical Virology 34:217-222, 1991.

5. Borhanmanesh $F$, Haghighi $P$, Herkmat $K$, Rezaizadeh K, Ghavami A. Viral hepatitis during pregnancy. Severity and effect on gestation. Gastrenterology 64:304-312, 1973.

6. Committee on Infectious Diseases. Universal Hepatitis B Immunization. Pediatrics 89:795-800, 1992.

7. Centers For Disease Control. Prevention of perinatal transmission of hepatitis $B$ virus. Prenatal screening of all pregnant women for hepatitis B surface antigen. Morbidity and Mortality Weekly Report 37:341-351, 1988.

8. Gonçalves CS, Pereira FEL, Vargas PRM, Ferreira LSE. Hepatocellular carcinoma HBsAg positive in pregnancy. Arquivo de Gastroenterologia, São Paulo 21:75-77, 1984.

9. Goudeau A, Lesage G, Denis F, Chiron JP, Yvonnet B, Barin F, Coursaget P, Mar DI. Lack of anti-HBc IgM in neonates with HBsAg carrier mothers argues against transplacental transmission of Hepatitis B virus infection. The Lancet 12:1103$1104,1983$.

10. Kumar ML, Dawson NV, Mc Cullough AJ, Radivoyevitch M, King K-C, Hertz R, Kiefer H, 
Cardoso DDP, Faria EL, Azevedo MSP, Queiroz DAO, Martins RMB, Souza TT, Daher RR, Martelli CMT. Soroepidemiologia para vírus da bepatite $B$ (VHB) em gestantes/parturientes e sua transmissão para recémnascidos em Goianina, GO. Revista da Sociedade Brasileira de Medicina Tropical 29:349-353, jul-ago, 1996.

Hampson M, Cassidy R, Tavill AS. Should all pregnant women be screened for hepatitis $B$ ? Annals of Internal Medicine 107:273-277, 1987.

11. Lange W, Masihi KN. Epidemiology and economic importance of hepatitis B in Federal Republic of Germany. Postgraduate Medical Journal 63 (suppl 2):21-26, 1987.

12. Lo K-J, Tsai Y-T, Lee S-D, Wu T-C, Wang J-Y, Chen G$\mathrm{H}$, Yeh C-L, Chiang BN, Yeh S-H, Goudeau A, Coursaget $\mathrm{P}$, Tong MJ. Immunoprophylaxis of infection with hepatitis $B$ virus in infants born to hepatitis B surface antigen-positive carrier mothers. The Journal of Infectious Diseases. 152:817-822, 1985.

13. Martelli CMT, Andrade ALSS, Cardoso DDP, Souza LCS, Silva SA, Sousa MA, Zicker F. Soroprevalência e fatores de risco para infecção pelo vírus da Hepatite B pelos marcadores AgHBs e antiHBs em prisioneiros e primodoadores de sangue. Revista de Saúde Pública de Sao Paulo 24:270-276, 1990.

14. Papaevangelou G, Roumeliotou-Karayannis A, Tassopoulos N, Kolaitis N, Stathopoulou P. Source of infection due to hepatitis B virus in Greece. The Journal of Infectious Diseases 147:987-989, 1981.

15. Porto SOB, Cardoso DDP, Queiroz DAO, Rosa H, Andrade ALSS, Zicker F, Martelli CMT. Prevalence and risk factors for $\mathrm{HBV}$ infection among street youth in Central Brazil. Journal of Adolescent Health 15:577-581, 1994.

16. Stevens CE, Toy PT, Tong MJ, Taylor PE, Vyas GN, Nair PV, Gudavalli M, Krugman S. Perinatal hepatitis B virus transmission in the United States. Prevention by passive-active immunization. Journal American Medical Association 253:22-29, 1985.

17.Tiollais P, Buendio M-A. Hepatitis B virus. Scientific American 4:48-54, 1991.

18. Voller A, Bartlett A, Bidwell DE. Enzime immunoassays with special reference to ELISA techniques. Journal of Clinical Pathology 31:507$520,1978$. 